\title{
The role of alpha oscillations in deriving and maintaining spatial relations in working memory
}

\author{
Kara J. Blacker ${ }^{1}$ - Akiko Ikkai ${ }^{1}$ Balaji M. Lakshmanan ${ }^{2}$. \\ Joshua B. Ewen ${ }^{1,2,3}$ • Susan M. Courtney ${ }^{1,4,5}$
}

Published online: 14 June 2016

(C) Psychonomic Society, Inc. 2016

\begin{abstract}
Previous research has demonstrated distinct neural correlates for maintenance of abstract, relational versus concrete, sensory information in working memory (WM). Storage of spatial relations in WM results in suppression of posterior sensory regions, which suggests that sensory information is task-irrelevant when relational representations are maintained in WM. However, the neural mechanisms by which abstract representations are derived from sensory information remain unclear. Here, using electroencephalography, we investigated the role of alpha oscillations in deriving spatial relations from a sensory stimulus and maintaining them in WM. Participants encoded two locations into WM, then after an initial maintenance period, a cue indicated whether to convert the spatial information to another sensory representation or to a relational representation. Results revealed that alpha power increased over posterior electrodes when sensory information was converted to a relational representation, but not when the
\end{abstract}

Electronic supplementary material The online version of this article (doi:10.3758/s13415-016-0439-y) contains supplementary material, which is available to authorized users.

Kara J. Blacker

kara.blacker@jhu.edu

1 Department of Psychological and Brain Sciences, Johns Hopkins University, 3400 N Charles St., Baltimore, MD 21218, USA

2 Neurology and Developmental Medicine, Kennedy Krieger Institute, Baltimore, MD, USA

3 Department of Neurology, Johns Hopkins University School of Medicine, Baltimore, MD, USA

4 Department of Neuroscience, Johns Hopkins University School of Medicine, Baltimore, MD, USA

5 F.M. Kirby Center, Kennedy Krieger Institute, Baltimore, MD, USA information was converted to another sensory representation. Further, alpha phase synchrony between posterior and frontal regions increased for relational compared to sensory trials during the maintenance period. These results demonstrate that maintaining spatial relations and locations in WM rely on distinct neural oscillatory patterns.

Keywords Abstract · EEG $\cdot$ Relational $\cdot$ Phase synchrony · Working memory

The ability to maintain and manipulate relevant information in working memory (WM) is a critical component of goaldirected and adaptive behavior. The vast majority of research has focused on WM for sensory-based information such as visual objects or spatial locations and the corresponding neural mechanisms by which sensory information is maintained in WM. For example, a body of work has described the role of the prefrontal cortex, (PFC; for reviews see Curtis \& D'Esposito, 2003; D’Esposito, 2007; Levy \& GoldmanRakic, 2000), posterior parietal cortex (PPC; e.g., Todd \& Marois, 2004), and primary visual cortex (e.g., Serences, Ester, Vogel, \& Awh, 2009) in the maintenance of visuospatial information in WM. However, sometimes it is necessary to derive and maintain abstract information in WM, such as relationships, rules, or strategies. This type of abstract, nonsensory information represents a critical component in one's ability to solve novel problems, flexibly guide goal-directed behavior, and to extract relevant information from a complex environment. The neural mechanisms underlying how abstract information is derived from sensory information and maintained in WM remains unclear.

Recent work has begun to suggest that storing concrete, sensory information in WM may be distinct from storing abstract, non-sensory information in WM. Previous single-cell 
recording studies have demonstrated that PFC and PPC contain neurons that represent a variety of different forms of abstract information, including rules (Wallis, Anderson, \& Miller, 2001), category membership (Freedman, Riesenhuber, Poggio, \& Miller, 2001; Swaminathan \& Freedman, 2012), strategies (Genovesio, Brasted, Mitz, \& Wise, 2005; Tsujimoto, Genovesio, \& Wise, 2012), and spatial relations (Chafee, Averbeck, \& Crowe, 2007), as well as sensory information across WM delays (Funahashi et al., 1989; Gnadt \& Andersen, 1988; Goldman-Rakic, 1995). More recently, functional magnetic resonance imaging (fMRI) evidence suggests that sub-regions of PPC and PFC are differentially active during maintenance of abstract versus object-specific information (Ackerman \& Courtney, 2012; Montojo \& Courtney, 2008). It is unclear, however, how this abstract information may be derived from sensory stimuli and whether these different types of information are in a hierarchical or competitive relationship in the brain.

Previous work within the sensory WM literature has demonstrated that suppression of competing, task-irrelevant information is crucial to effective performance due to the capacitylimited nature of WM (Kuo, Stokes, \& Nobre, 2012; Rutman, Clapp, Chadick, \& Gazzaley, 2010; Vogel, McCullough, \& Machizawa, 2005). Accumulating evidence suggests that neural oscillations are important in both the maintenance of relevant and selective suppression of irrelevant sensory information in WM, such as object locations and features (Medendorp et al., 2007; for a review see, Roux \& Uhlhaas, 2013). Specifically, oscillations in the alpha frequency band (8$13 \mathrm{~Hz}$ ) have been particularly well documented in human electroencephalography (EEG). Maintenance of information in $\mathrm{WM}$ is associated with increases in alpha power over posterior regions, which is thought to reflect suppression of incoming sensory input that would interfere with the currently maintained information (Jensen, Gelfand, Kounios, \& Lisman, 2002; Klimesch, Doppelmayr, Schwaiger, Auinger, \& Winkler, 1999; Klimesch, Sauseng, \& Hanslmayr, 2007; Krause, Lang, Laine, Kuusisto, \& Porn, 1996). Although alpha was originally thought to reflect cortical idling (Adrian \& Matthews, 1934; Pfurtscheller, Stancak, \& Neuper, 1996), more recent evidence suggests that alpha oscillations may have a more direct role in selective attention and WM, particularly in the suppression of task-irrelevant brain regions (Bengson, Mangun, \& Mazaheri, 2012; Fu et al., 2001; Jensen \& Mazaheri, 2010; Jokisch \& Jensen, 2007; Kelly, Lalor, Reilly, \& Foxe, 2006; Klimesch et al., 2007; Rihs, Michel, \& Thut, 2007; Sauseng et al., 2009; van Dijk, van der Werf, Mazaheri, Medendorp, \& Jensen, 2010; Worden, Foxe, Wang, \& Simpson, 2000). For example, Fu et al. (2001) had participants perform either an auditory or a visual discrimination task and found increased alpha power over parieto-occipital sites when attention was cued to the auditory modality compared to the visual modality. The authors suggested that these parietal regions are capable of integrating sensory cues in order to control the deployment of visual attention. Thus, alpha oscillations appear to reflect a mechanism by which brain regions that represent task-irrelevant information are suppressed in order to prioritize task-relevant information processing (Jensen \& Mazaheri, 2010; Kelly et al., 2006).

When abstract, non-sensory information is maintained in $\mathrm{WM}$, the original sensory representation may become taskirrelevant and may even interfere or compete with the to-bemaintained abstract information. Therefore, alpha oscillations may represent the mechanism by which the irrelevant, sensory information is suppressed when abstract information needs to be maintained in WM. One previous study has examined fluctuations in alpha oscillations in response to maintaining abstract versus sensory information in WM. Ikkai, Blacker, Lakshmanan, Ewen, and Courtney (2014) had participants encode and maintain either concrete spatial coordinates or abstract spatial relations of objects and found that maintaining abstract relations resulted in increased alpha power over posterior electrode sites. Furthermore, there was evidence for weaker alpha lateralization in response to a covert shift of attention to one visual hemifield during storage of abstract, relational information as compared to concrete, sensory information. These results suggested that sensory cortex is suppressed during WM for spatial relations relative to WM for absolute spatial coordinates.

It remained unclear, however, whether this effect on sensory cortex was due specifically to WM for abstract information or whether the oscillatory activity was related to the initial processing of the sensory stimulus. In our previous study by Ikkai et al. (2014), participants completed the two types of WM tasks in a blocked design, which could have allowed for use of a pre-determined encoding strategy depending on whether a spatial location or relation was relevant in a given block. Even though the previous study demonstrated that the alpha power increase continued throughout the maintenance period, suggesting sensory suppression is an important factor in maintenance of spatial relations in WM, it could have instead reflected a continuation of that initial stimulus encoding state in anticipation of the processing of the test stimulus. In the current study, therefore, we separated the initial stimulus encoding period from the transformation/manipulation of that sensory representation into a spatial relationship and the subsequent maintenance of abstract information. This novel design enabled examination of the role that alpha oscillations play in explicitly deriving abstract spatial relations from a sensory stimulus and subsequently maintaining them in WM. The current task design (see Methods) required participants to always initially encode and maintain two sensorybased spatial locations. Then a cue instructed participants to manipulate the sensory information held in WM into either a relative spatial relation or a specific, retinotopic spatial 
location and maintain that information over a second delay period before comparing it to a test stimulus. We predicted that deriving an abstract relationship from a sensory WM representation would result in greater suppression of sensory regions (indicated by more posterior alpha power) as compared to when another concrete, sensory location was derived from the same maintained sensory information. This comparison to a sensory WM task that also required derivation of a new representation that was not a direct copy of the initial sample stimulus further ensured that any differences in neural activity observed would be due to the type of information, rather than the presence or absence of a change from sample stimulus to WM representation.

Finally, we were interested in the potential role that frontal regions play in this derivation and maintenance process, which our novel task design here allowed us to examine. While there is much evidence to support the hypothesis that alpha oscillations over posterior regions are indicative of suppression or inhibition of those sensory regions, less work has examined the role that other brain regions may have on modulating that oscillatory-based suppression. For example, it has been proposed that sensory WM relies on connections between PFC and domain-specific sensory regions (Cohen, Sreenivasan, \& D'Esposito, 2014; Courtney, Ungerleider, Keil, \& Haxby, 1997; Fuster, 2000; Smith \& Jonides, 1999; Ungerleider, Courtney, \& Haxby, 1998). In line with this evidence, previous studies have suggested that PFC plays a causal role in modulating posterior alpha during WM (Sauseng et al., 2005; Zanto, Rubens, Thangavel, \& Gazzaley, 2011). Moreover alpha phase synchrony between frontal and sensory regions has been suggested to play a role in the inhibition of anticipated, task-irrelevant stimuli (Bonnefond \& Jensen, 2012; Sadaghiani et al., 2012). Therefore, in the current study we examined the role that oscillatory activity in frontal regions plays when relational versus sensory information is maintained in WM. If frontal regions are involved in modulating posterior alpha power over sensory regions, then we predicted that deriving and maintaining an abstract relationship in WM would elicit greater frontal-posterior alpha phase synchrony, as compared to maintaining concrete, sensory information in WM. We also predicted that both alpha power and synchrony would evolve together across time as these WM representations were derived during the course of the task.

\section{Methods}

\section{Participants}

Twenty-five neurologically healthy adults (10 male, 18-31 years of age) participated and were compensated monetarily. All participants had normal or corrected-to-normal visual acuity (based on self-report), and gave written informed consent approved by the Institutional Review Boards of Johns Hopkins University and the Johns Hopkins Medical Institutions. Six participants were excluded from any analyses due to excessive EEG artifacts and/or incorrect trials, leaving our final sample to be analyzed as $n=18$.

\section{Task and procedures}

Experimental stimuli were controlled by MATLAB (The MathWorks, Natick, MA, USA) using Psychophysics Toolbox extensions (Brainard, 1997; Pelli, 1997), and displayed on a Dell LCD monitor. Participants were seated $92 \mathrm{~cm}$ away from the monitor, and given a Logitech game controller to enter responses.

As shown in Fig. 1, a trial began with a white fixation square $\left(0.1^{\circ}\right.$ of visual angle) appearing in the center of the display for $1,000 \mathrm{~ms}$. Next, a memory array containing two circles of varying sizes each subtending between $0.35-0.8^{\circ}$ of visual angle, one with a red center and one with a green center where the center subtended $0.1^{\circ}$ of visual angle, appeared for $500 \mathrm{~ms}$. The memory array always contained one circle in the left hemifield and one circle in the right hemifield. The vertical distance between circles, center to center, was $1.5-4.3^{\circ}$ of visual angle. Participants were instructed to remember the locations of the two circles. After a 2,000-ms delay period (Delay 1), a 200-ms tone was presented via headphones. The tone was a high-pitch long tone, a low-pitch long tone, two high-pitch short tones, or two low-pitch short tones. The length of the long tone was identical to the combined length of the two short tones together. All tone types were counterbalanced across trial types (i.e., four possible combinations of tone type with trial type). The single or double tone indicated whether the trial was a "Location" or "Relation" trial and the pitch of the tone indicated which circle (greencenter/red-center) was the reference. For example, a long tone would indicate a Location trial and two short tones would indicate a Relation trial. The pitch of the tone(s) would either be high or low. A high pitch long tone would indicate that the participant should remember the exact vertical location of the red-center circle, by drawing an imaginary horizontal line through its location. Alternatively, a low pitch long tone would indicate that the vertical location of the green-center circle should be remembered. If participants were cued for a Relation trial with two short tones, those tones would again either be high or low pitch. Two short, high-pitch tones would indicate that the participant should remember the red-center circle relative to the green-center circle in terms of relative vertical location (e.g., the green is the reference and the red is above/below the green). Alternatively, two short, low-pitch tones would indicate that the green-center circle should be remembered relative to the red-center circle (e.g., the red is the reference, the green is above/below the red). The instructional meanings of long versus short and high versus low pitch 


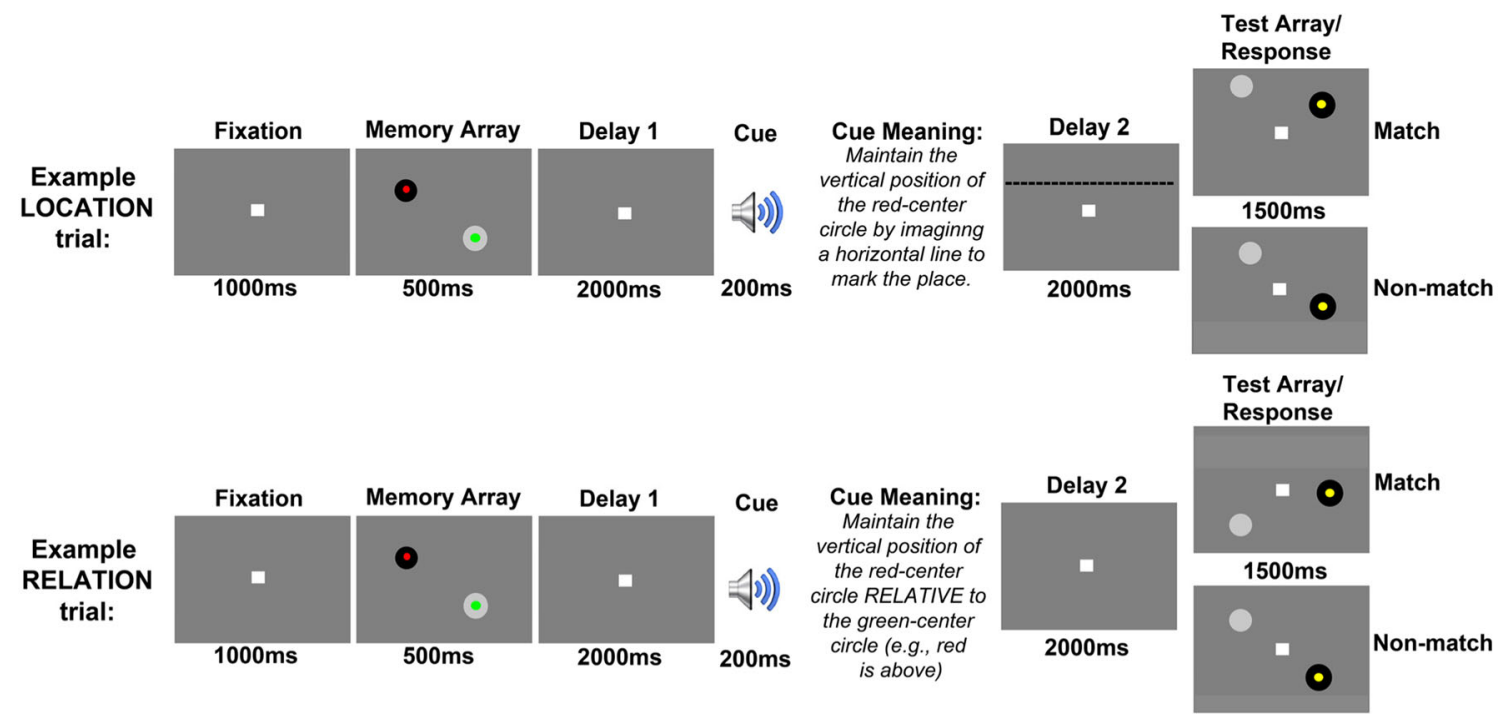

Fig. 1 Example trial schematics. Both types of trials began identically with a memory array containing two colored circles, and participants were instructed to remember the locations of those two circles. After an initial delay (Delay 1), an auditory cue indicated whether a trial was a Relation or Location trial. The cue also indicated which memory array item was tobe-remembered. Cue meanings were counterbalanced across participants. For Location trials (above), participants were instructed to remember the vertical position of the cued circle by imagining a horizontal line to mark

tones (i.e., two tone lengths, two pitches) for each trial type and reference color (i.e., red-reference Location, greenreference Location, red-reference Relation, green-reference Relation) were counterbalanced across participants.

After this auditory cue was presented, participants had to maintain the relevant information over a subsequent 2,000-ms delay period (Delay 2). Finally, a 1,500-ms test array would appear with two circles, one of which would contain a yellow center. For Location trials, participants were asked to indicate whether the yellow-center circle had the same vertical location as the cued memory circle (i.e., is the yellow-center circle on the imaginary horizontal line held in memory?). For nonmatch Location trials, the yellow-center circle's vertical position was a minimum of $1.9^{\circ}$ of visual angle from the vertical position of the cued sample array circle. For Relation trials, the solid circle acted as the reference for judging whether the test array matched the relationship held in memory. For example, if the participant was cued to remember that green was above the reference, red-center circle, and if the yellow-center test circle was above the solid, reference circle, then a match response was required. In sum, participants were asked to encode the two locations into WM and maintain them over an initial delay period (Delay 1), and then were cued to convert those locations into either one concrete, sensory representation or one abstract, relational representation, then maintain that information over a second delay period (Delay 2) before comparing the relevant WM information to the corresponding type of information in a test array. the place (line shown here only for illustration). At test, participants judged if the yellow-center circle was in the same vertical position as the cued circle. For Relation trials (below) participants were instructed to remember the vertical position of the cued circle relative to the other sample circle (e.g., red is above). At test, participants judged whether the yellow-center circle had the same relationship to the gray test circle as the sample circles had to each other

Importantly, this task design ensured that participants could not anticipate, and thus direct attentional resources to, any particular sensory representation of the upcoming test stimulus in order to perform the Relation task. Further, the absolute location of the yellow-center test circle for Relation trials was not predictive of the correct response (i.e., if participants' performed all Relation trials as Location trials, performance would be at chance). Similarly, the relational information alone could not predict the correct response for Location trials. Further, the horizontal position of the sample circles was not predictive of the correct response for either trial type (i.e., if participants had simply maintained the horizontal position for Location trials and made their response based on that information as opposed to the vertical position, accuracy would have been at chance). This design encouraged participants to discard the irrelevant location information for Relation trials and the irrelevant relational information for Location trials. In this sense, the task encouraged participants to maintain in WM a representation of the derived relation or derived location but not both, because these two types of information would be in "competition" with one another regarding control over the behavioral response.

For both trial types, $50 \%$ of trials were match trials and $50 \%$ were non-match trials. Response keys for match and non-match were counterbalanced across participants. The inter-trial interval (ITI) was chosen randomly for each trial from 1,250 to $1,500 \mathrm{~ms}$ in $50-\mathrm{ms}$ increments, during which the fixation square was black. 
While EEG was recorded, participants completed a total of 256 trials (128 Location, 128 Relation randomly intermixed), which were broken down into eight runs of 32 trials. Prior to the EEG session, on a separate day, participants completed 128 practice trials. One participant was not invited to participate in the EEG session due to poor performance $(<50 \%$ accuracy).

\section{Data collection and analysis procedures}

EEG recording EEG data were recorded at 128 sites covering the whole scalp with approximately uniform density using an elastic electrode cap referenced to the average of all electrodes during recording (WaveGuard cap with 128-channel Duke, equidistant electrode placement, layout: Advanced Neuro Technology, The Netherlands). Electrode impedance was kept below $10 \mathrm{k} \Omega$. All EEG electrodes were recorded continuously in DC mode at a sampling rate of $512 \mathrm{~Hz}$ using an anti-aliasing filter with a 138-Hz cutoff and a high-impedance ANT WaveGuard amplifier.

Preprocessing Data were analyzed using the Fieldtrip software package (Oostenveld, Fries, Maris, \& Schoffelen, 2011). Data were first high-pass filtered at $1 \mathrm{~Hz}$, and then segmented into epochs covering the time from $2.0 \mathrm{~s}$ before to $9.0 \mathrm{~s}$ after the onset of the fixation display in each trial. Depending on the length of the randomly chosen ITI (i.e., 1,250-1,500 ms), a trial ranged in length from 8.45 to $8.7 \mathrm{~s}$. We chose epochs from -2.0 to $9.0 \mathrm{~s}$ from the onset of fixation to ensure that the final epoch of a trial captured activity that lasted throughout the test period with sufficient time afterward to allow for analysis of the alpha frequency band with the sliding time windows (see Spectral analysis below for details). Importantly, the chosen epochs never included the next trial's sample array. Independent components analysis (ICA) was performed on the epoched data, and the eye blink component was identified and removed for each participant's data. After eye blink correction, EEG waveforms from frontal electrodes (i.e., RE1/LE1) were visually inspected to identify voltage fluctuations (i.e., fluctuation greater than $18.75 \mu \mathrm{V}$ or less than $-18.75 \mu \mathrm{V})$ typical of eye movements. Trials containing horizontal eye movements were rejected entirely. EEG data were analyzed only for correct trials. To maintain sufficient statistical power for each trial type, six participants with more than $30 \%$ trial rejection due to any combination of eye-blink or eye-movement artifacts, or behavioral errors were excluded, leaving a final sample of 18 . These 18 participants had an average of 101 Location trials $(S D=8)$ and an average of 109 Relation trials $(S D=9)$ after incorrect trials and trials with artifacts were removed.

Spectral analysis Power spectra were calculated using a timefrequency transformation based on multiplication in the frequency domain from 1 to $30 \mathrm{~Hz}$ with $0.5-\mathrm{Hz}$ increments using a hanning taper applied to short sliding time windows (Percival \& Walden, 1993) every $100 \mathrm{~ms}$. An adaptive time window of five cycles for each frequency $(\Delta \mathrm{T}=5 / f)$ was applied.

Statistical analysis To obtain statistics corrected for multiple comparisons we used a nonparametric randomization test (Maris \& Oostenveld, 2007; Nichols \& Holmes, 2002) to statistically test for differences between Relation and Location trial types. This procedure controls for Type I error by calculating the cluster-level statistics by randomizing trial labels at each iteration. First, spectral data from each of the 128 electrodes across the scalp were averaged over the time and frequency range of interest. Our frequency range of interest was alpha $(8-13 \mathrm{~Hz})$ based on previous work described above in the Introduction. Our time range of interest was the Delay 2 period (i.e., 3.7-5.7 $\mathrm{s}$ after the onset of fixation), but we excluded the first 500ms of the Delay 2 period because this time period likely contained sensory-evoked response activity from the cue stimuli (e.g., van Gerven et al., 2009; for a more detailed discussion of this topic, also see Bastiaansen, Mazaheri, \& Jensen, 2012). Next, a $t$-value was calculated at each electrode. For each iteration randomizing trial labels, clusters of electrodes where the alpha-level was $<0.05$ were identified, and their $t$-values were summed. The largest sum of $t$-values was used as a $t$ statistic. This procedure was repeated 5,000 times to create the null distribution. The $p$-value was estimated according to the proportion of the null distributions exceeding the observed cluster-level $t$-statistic. Based on these results, we then selected any significant clusters of electrodes to plot full time-frequency representations (TFR) for the entire trial length and all low frequency bands $(1-30 \mathrm{~Hz})$.

Phase-locking values analysis To investigate alpha phase synchrony, a method suggested by Lachaux, Rodriguez, Martinerie, and Varela (1999), termed phase-locking value (PLV), was used. PLVs represent the phase covariance between two signals that are close in time. Unlike the more traditional method of spectral coherence, PLVs separate the phase and amplitude components, which makes PLV far less susceptible to the amplitude of the signal, and can be directly interpreted in the framework of neural integration (Lachaux et al., 1999). Phase-locking between two signals $\left(s_{i}^{a}\right.$ and $\left.s_{i}^{b}\right)$ was quantified, from the unaveraged signals, using wavelet analysis (Lachaux et al., 1999). A complex representation of the phase for trial $i$ at time $t$ and frequency $f_{0}$ is given by the convolution of a Morelet wavelet centered at $10.5 \mathrm{~Hz}$ (i.e., center of the alpha range), 
$w\left(t, f_{0}\right)=\mathrm{A} \exp \left(-t^{2} / 2 \sigma_{t}^{2}\right) \exp \left(j 2 \pi f_{0} t\right)$, and the signal $s_{i}^{a}$ normalized by the amplitude

$\Phi_{i}^{a}\left(t, f_{0}\right)=\frac{w\left(t, f_{0}\right) * s_{i}^{a}(t)}{\left|w\left(t, f_{0}\right) * s_{i}^{a}(t)\right|}$

The width of the wavelet $\left(m=f_{0} / \sigma_{f}\right)$ was chosen to be 7 (Grossman, Kronland-Martinet, \& Morlet, 1989); where $\sigma_{f}=1 / 2 \pi \sigma_{t}$. The PLVs over $N$ trials between signals $s_{i}^{a}$ and $s_{i}^{b}$ are defined as:

$P L V\left(t, f_{0}\right)=\frac{1}{N} \sum_{i=1}^{N}\left(\Phi_{i}^{a} / \Phi_{i}^{b}\right)$

PLVs range from 0 to 1 , which estimates the variability of phase differences between two signals across trials. If the phase difference varies little across trials, PLV is close to 1 ; with large variability in the phase difference it is close to zero. To stabilize the variance of the PLV data, an inverse hyperbolic tangent transformation was used (Hummel \& Gerloff, 2005). For all PLV calculations, we selected RC7 (i.e., a right posterior electrode site) as the seed electrode. We examined average alpha power $(8-13 \mathrm{~Hz})$ for each trial type and selected the electrode that showed the greatest power modulation during the delay period for Location and for Relation separately. RC7 showed the greatest alpha delay power for both trial types and was therefore chosen as the seed for the PLV analysis. ${ }^{1}$ PLV between this seed and each of the other electrodes was calculated separately for Relation and Location trials.

To statistically compare alpha phase synchrony between the two trial types, we used a nonparametric randomization test, similar to that used to compare spectral power between trial types. Specifically, PLVs for each trial type were averaged across the time period of interest (i.e., Delay 2). A $t$-value was then calculated for each electrode across the scalp (except the seed), with trial labels randomized. For each iteration, clusters of electrodes where the alpha-level was $<0.05$ were identified, and their $t$-values were summed. The largest sum of $t$-values was used as a $t$-statistic. This procedure was repeated 5,000 times to create the null distribution. The $p$-value for a cluster with correct trial labels was then estimated according to the proportion of the null distributions exceeding the observed cluster-level $t$-statistic.

Scalp current density (SCD) control analysis To ensure our PLV estimates were not due to volume conduction, we applied a Scalp Current Density (SCD) transformation and ran a control analysis. EEG data were converted into SCD distributions, computing the second spatial derivative (the surface

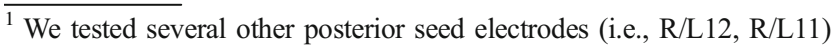
and found similar results in terms of significance and directionality of effect; therefore we only present the results from RC7.
}

Laplacian) of the interpolated potential distribution (Perrin, Pernier, Bertrand, \& Echallier, 1989).

Whole trial spectral and PLV plots The time period of interest here was Delay 2, which is why the statistical analyses (detailed above) only test for differences between Relation and Location trials during this time period. However, for completeness we also provide plots of alpha power and PLV for the entire trial duration. Using the Fixation period as a baseline, we calculated corrected [(signal - baseline)/baseline] alpha power $(8-13 \mathrm{H} \mathrm{z})$ for every $100 \mathrm{~ms}$ time bin for each participant and plotted the average across participants and across electrodes of interest (see Results for details). To plot out PLVs for the entire trial duration, we calculated PLV (see Phase-locking values analysis above) between the seed electrode (RC7) and those frontal electrodes that showed significant differences between Relation and Location trials for every $100-\mathrm{ms}$ time bin for each participant. These whole trial illustrations are shown in Figs. 3D/E and 4B. No statistical analyses were tested on the entire trial duration data; these plots simply show the fluctuation of spectral alpha power and phase synchrony throughout the entire trial for Relation and Location trials separately.

\section{Results}

\section{Behavioral results}

Six participants were excluded from any analysis due to having $30 \%$ or more of trials eliminated from artifacts and/or incorrect responses. On the final sample of 18 participants, we tested for differences in mean accuracy and response time (RT) between Location and Relation trials using pairedsamples $t$-tests. For RT, there was no significant difference between trial types, $t(17)=1.6, p>0.1$. For accuracy, a significant difference between trial types did emerge, $t(17)=6.15$, $p<0.001$, with higher accuracy for Relation trials compared to Location trials (Fig. 2). Despite this difference in accuracy, performance was quite high in both trial types (above $90 \%$ ). Because there were so few trials with errors, we were not able to compare EEG data for correct versus incorrect trials. EEG data were analyzed only for correct trials. While these results suggest a difference in difficulty between the two trial types, the most important feature of our design is that in both trial types participants were asked to derive either one spatial location or one spatial relation from identical sensory information held in WM, keeping the WM load nominally the same across trial types, and this comparison is the focus of our EEG analyses. However, we will address this disparity in accuracy further in the Discussion section. 

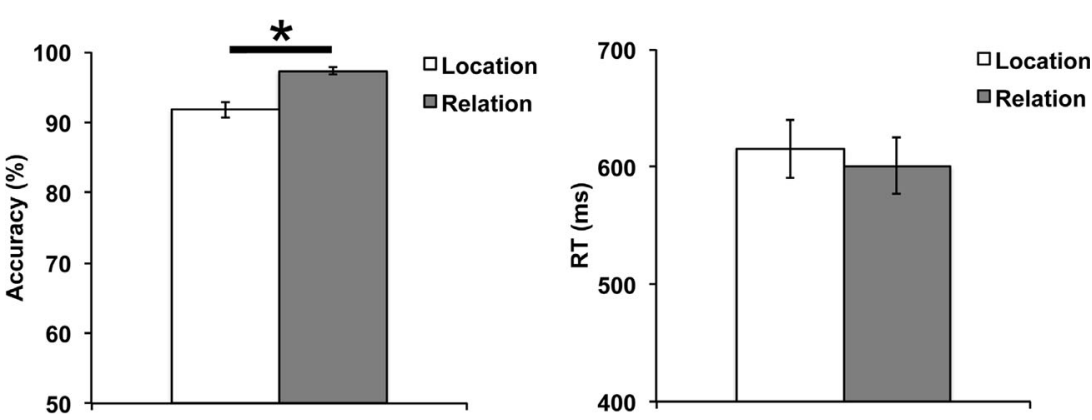

Fig. 2 Behavioral results for accuracy and response time (RT). Error bars represent standard error of the mean. ${ }^{*} p<0.001$

\section{Alpha power: Relation versus location trials}

First, we contrasted raw power between Relation and Location trials by calculating a Task Modulation Index (TMI): $($ Relation - Location)/(Relation + Location). A positive TMI would result from relatively higher power in Relation compared to Location trials (i.e., warmer colors in Fig. 3). Alternatively, a negative TMI would result from relatively higher power in Location than Relation trials (i.e., cooler colors in Fig. 3). We focused our analyses on the Delay 2 period and in the alpha frequency band $(8-13 \mathrm{~Hz})$ by using a nonparametric randomization test to statistically test TMI versus 0 across all 128 electrodes. As shown in Fig. 3A, there were three significant clusters of electrodes showing greater alpha power for Relation compared to Location trials during Delay 2: A large cluster of posterior electrodes, $p<0.0005$, a left frontal cluster, $p<0.005$, and a single right frontal electrode site, $p<0.01$. Next, we used these significant electrode clusters to visualize the dynamics of the full power spectra in both the time and frequency domains. Figure 3B illustrates the TFR for the significant cluster of posterior electrodes and Fig. 3C illustrates the TFR for the two significant frontal electrode clusters taken together. As expected from the way these electrode clusters were selected, both TFR show increased alpha band power during the Delay 2 period for Relation compared to Location trials. For both the posterior and frontal clusters, we ran a secondary randomization test on the entire trial duration and all low frequency bands (1$30 \mathrm{~Hz}$ ). Supplemental Fig. A shows a TFR of these resulting $p$-values, which demonstrates that the significant time-frequency points are almost entirely limited to the alpha frequency band. The posterior alpha modulation during the Delay 2 period is consistent with the previous study by Ikkai et al. (2014), and the implications for these results within the previously proposed inhibition hypothesis of alpha are detailed in the Discussion. Furthermore, these results with the Relation/Location instruction cue following Delay 1 also demonstrates that the posterior alpha modulation is evident after information in WM is manipulated, not only from a different initial encoding of the stimulus information into WM.

There are two novel findings that emerge from these results as well: (1) bilateral frontal electrodes showed a similar pattern of alpha modulation as the posterior electrodes, and (2) the TFR illustrates more alpha power for Relation trials compared to Location trials during the Test period as well as the Delay 2 period. Figures 3D and E show the time course of alpha power for the Relation and Location trials separately. Alpha power gradually increased for both trial types during Delay 1, as would be expected because the trial types are identical during that period. ${ }^{2}$ This increase in alpha power is consistent with previous work demonstrating that WM maintenance is associated with more alpha power over posterior sites (Jensen et al., 2002; Klimesch et al., 1999; Krause et al., 1996). After the cue that indicated what information was to be derived and maintained during Delay 2, there was a rapid decline in alpha power for both trial types. This decline could indicate a return to baseline or represent the decrease in WM load resulting from the conversion of the WM information from two locations to either one horizontal line or one spatial relationship, which would be consistent with studies that show that alpha power is modulated with WM load (Jensen et al., 2002; Manza, Hau, \& Leung, 2014). Approximately $500 \mathrm{~ms}$ after the cue, however, the alpha power for the Relation trials increased while the power for the Location trials did not. We explored each of these findings in turn with further analyses.

Given the significant difference in behavioral accuracy between the two trial types, one could argue that Location trials were more difficult or involved a higher memory load, which resulted in less alpha power compared to Relation trials. If this were the case, one would expect a relationship between

\footnotetext{
${ }^{2}$ We statistically confirmed that there was no difference between Relation and Location trials prior to the cue in Delay 1 by using a nonparametric randomization test comparing TMI vs. 0, which yielded no significant clusters. The similarity between trial types in Delay 1 can be visualized in Fig. 3.
} 


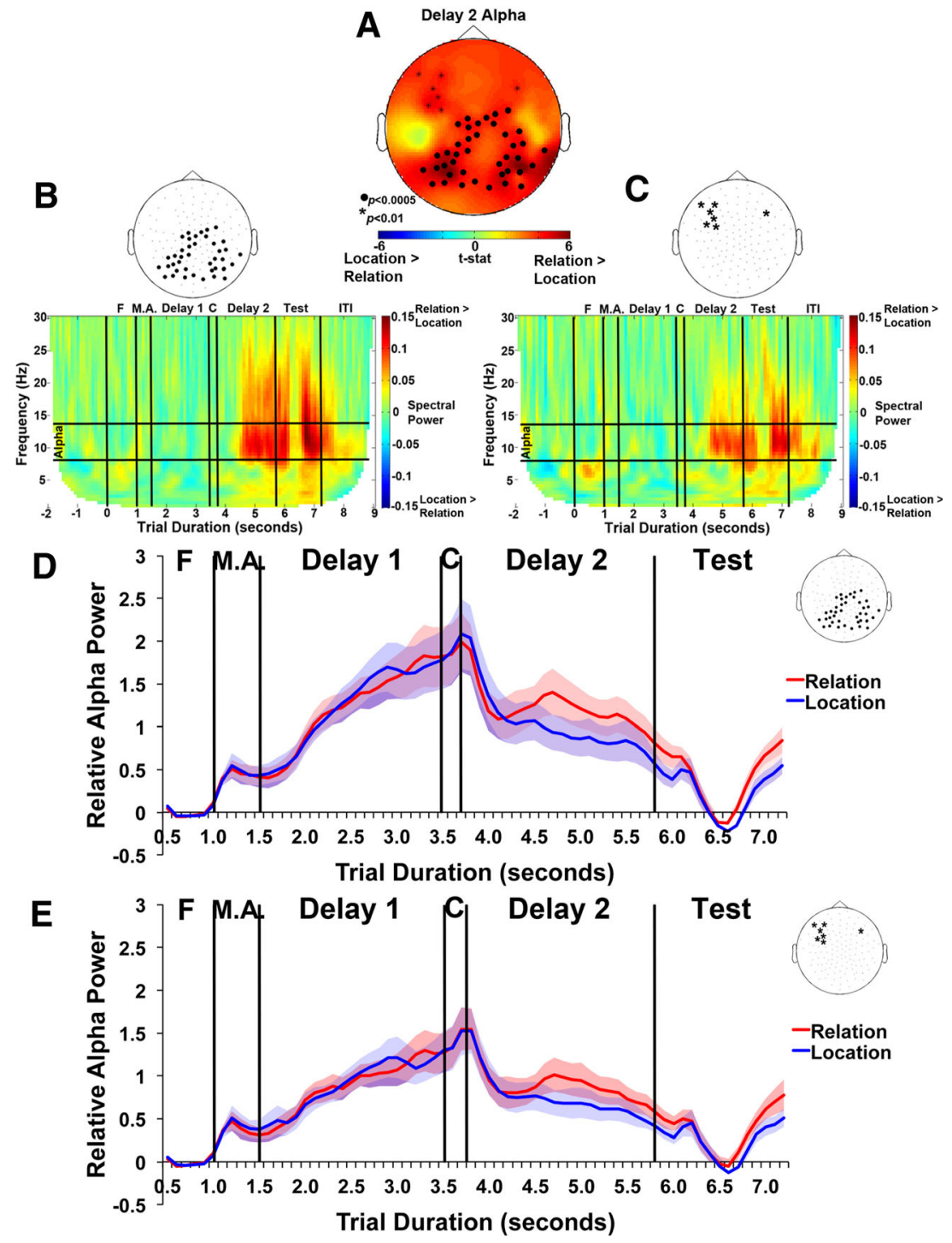

Fig. 3 (A) Results from a nonparametric randomization test comparing Relation vs. Location for Delay 2 period alpha. (B) Significant posterior electrodes and their corresponding time-frequency representations (TFR). (C) Significant frontal electrodes and their corresponding TFR. Warmer colors indicate more alpha for Relation trials compared to Location trials and marked electrodes represent statistically significant clusters. (D/E)
Baseline-corrected alpha power for Location and Relation trials separately across the entire trial duration. Shown for significant (D) posterior electrode cluster and (E) anterior electrode clusters. Shaded bands represent between-subject standard error of the mean. Each trial event is marked with vertical lines: Fixation (F), Memory Array (M.A.), Delay 1, Cue (C), Delay 2, Test and ITI accuracy and alpha power regardless of trial type. Therefore we tested a correlation analysis between task accuracy and raw alpha power at the significant posterior and frontal electrode clusters for the Delay 2 period (minus the first $500 \mathrm{~ms}$ ). However, this analysis revealed no significant relationship between task accuracy averaged across both trial types and posterior $(R=0.12, p=0.49)$ or frontal $(R=-$ $0.12, p=0.48$ ) alpha power. Accuracy was also not correlated with frontal or posterior alpha power within Location trials $(p \mathrm{~s} \geq 0.46)$ or within Relation trials $(p \mathrm{~s} \geq 0.44)$ separately. This lack of a correlation shows that the difference in accuracy level for the two trial types cannot readily explain the increased alpha power for Relation trials compared to Location trials.

\section{Alpha phase synchrony}

To better understand the origin and control of these dynamic modulations of alpha power, we tested PLVs between posterior and frontal electrode sites for differences in alpha phase synchrony between the two trial types. We calculated PLVs for Location and Relation trials separately for the Delay 2 period. As described above, we chose RC7, a right posterior electrode, as the seed electrode, because it had the greatest 
alpha power modulation for both trial types. In order to test for differences in PLV between our two trial types, we used a nonparametric randomization test across all electrodes (except for the seed electrode).

During the Delay 2 period, there was significantly greater alpha phase synchrony for Relation trials compared to Location trials between the seed electrode and a cluster of frontal electrodes, $p<0.01$ (see Fig. 4A). We also plotted alpha phase synchrony across the entire trial duration (shown in Fig. 4B), by averaging PLVs between the right posterior seed electrode (RC7) and all the electrodes in this cluster. As an additional visualization, the phase lag between the seed electrode and the significant cluster of frontal electrodes for each participant for the Delay 2 period is shown for Relation and Location trials in Supplemental Fig. B. Note that the frontal electrodes demonstrating greater alpha phase synchrony are different from the frontal electrodes demonstrating increased alpha power. Together, these results demonstrate that both alpha power and alpha phase synchrony are dynamically modulated across time during WM maintenance, manipulation, and test stimulus periods.

\section{SCD control analysis}

The alpha phase synchrony here exemplifies long-range synchrony, which is less susceptible to overinflated PLV estimates due to volume conduction (Lachaux et al., 1999), as compared to shorter-range synchronization. However, to ensure our PLV estimates were not due to volume conduction, we applied a SCD transformation (Perrin, et al., 1989) and retested our analysis above. With the SCD transformation, there were significantly greater PLVs for Relation compared to Location between the RC7 seed electrode and a cluster of right-central electrodes, $p=0.01$, and a cluster of right frontal electrodes, $p<0.05$ (see Supplemental Fig. C). While the topography was slightly different after the SCD transformation, the direction of the effect and the significance were unchanged from that reported above without the transformation, which suggests that our difference in PLV between Relation and Location trials is not a spurious result due to volume conduction. We also tested the same TMI versus 0 alpha power analysis with the SCD transformation applied, which yielded only the posterior cluster of electrodes showing significantly more alpha power for Relation trials compared to Location trials, $p=0.0001$ (see Supplemental Fig. C).

\section{Test period alpha power}

Examination of the full TFR comparing Relation versus Location revealed increased alpha power during the Test period for Relation compared to Location trials. This pattern, shown in Fig. 3, emerged for both the group of posterior and the group of frontal electrodes that were significantly modulated during the Delay 2 period. The time courses of alpha

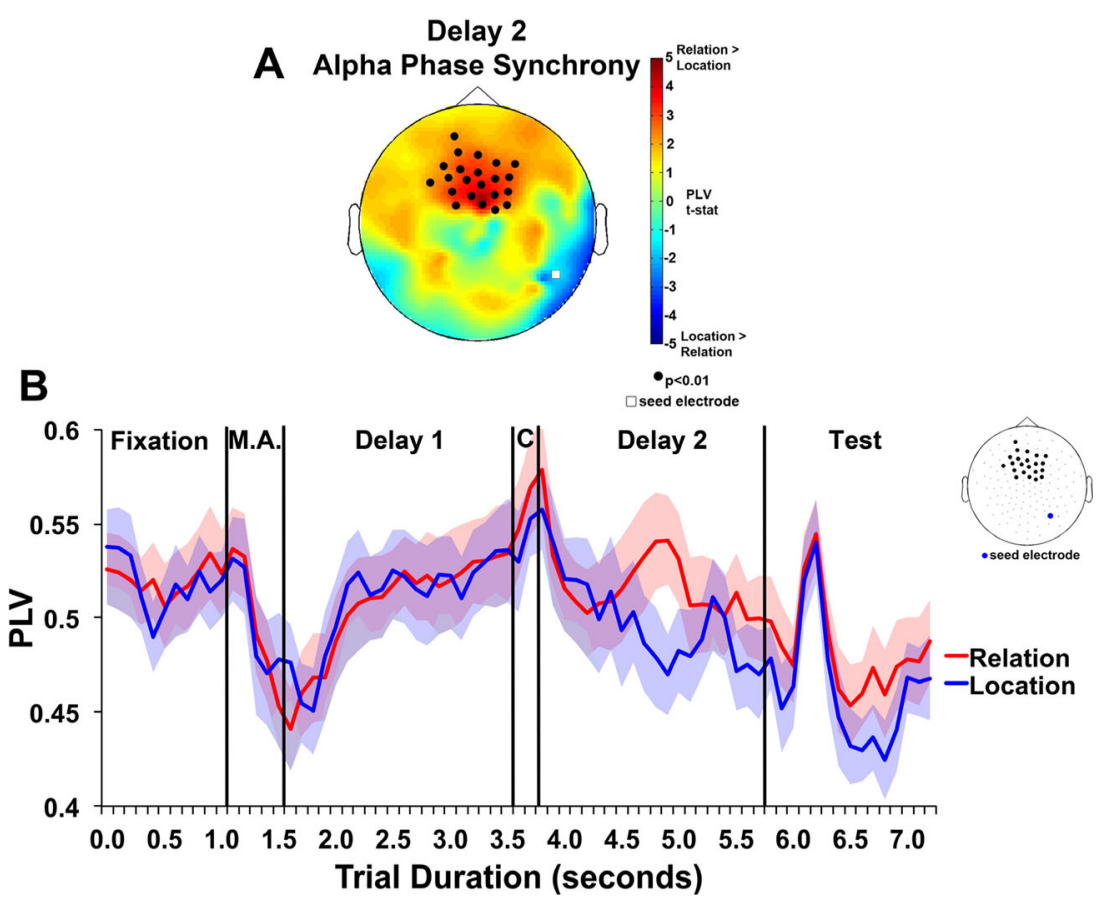

Fig. 4 (A) Results of a nonparametric randomization test comparing phase-locking values (PLVs) for Relation vs. Location trials. Warmer colors indicate greater PLVs for Relation compared to Location trials and marked electrodes indicate a cluster with significantly greater PLVs with the seed electrode for Relation compared to Location. (B) PLVs for
Relation and Location trials separately between a right posterior seed and significant frontal electrodes from the Delay 2 randomization test. Each trial event is marked with vertical lines: Fixation, Memory Array (M.A.), Delay 1, Cue (C), Delay 2, and Test. Shaded bands represent betweensubject standard error of the mean 
power and synchrony shown in Figs. 3 and 4, respectively, indicate a large spike in synchrony that is equivalent for both Location and Relation trials, followed by greater power and synchrony for the Relation trials. To evaluate whether this difference in alpha power extended to electrodes other than those identified in the Delay 2 period analysis, we examined alpha power during the test period (i.e., $500-1,500 \mathrm{~ms}$ after presentation of the test stimuli) using a similar nonparametric randomization test to statistically compare TMI versus 0 across all 128 electrodes. One large cluster of electrodes spanning most of the scalp yielded significantly more alpha power for Relation compared to Location trials, $p<0.01$ (see Supplemental Fig. D). The lack of spatial specificity of these results makes them difficult to interpret, but we will elaborate on potential interpretations in the Discussion section below.

\section{Discussion}

The current study sought to examine the role of posterior and frontal alpha oscillations in deriving abstract spatial relations from sensory-based information in WM. The task design allowed us to examine the specific oscillatory activity associated with manipulating a maintained sensory representation into an abstract relationship. When participants were required to derive an abstract spatial relation from the maintained sensory information there was increased alpha power over posterior regions. Further, maintaining a spatial relation in WM was associated with increased frontal alpha power and increased frontal-posterior alpha phase synchrony, which suggests that long-range alpha synchronization between frontal and posterior regions may play a crucial role in deriving and maintaining abstract information in WM. Together, these results suggest that alpha oscillations and their synchronization may be involved in controlling abstract versus sensory information processing.

An extensive literature has accumulated on the neural basis of maintaining sensory information in WM, but relatively little is known about how other types of information, such as abstract, non-sensory relations, are encoded, maintained, and used in WM. Abstract, non-sensory information such as relationships, rules, and strategies represent a critical component in one's ability to solve novel problems, apply previous knowledge to current goal-directed behavior, and to extract relevant information from a complex environment. The current study and other recent evidence suggest that WM for nonsensory information, such as abstract spatial relations, is distinct from WM for sensory-based information (Ackerman \& Courtney, 2012; Ikkai et al., 2014; Montojo \& Courtney, 2008).

A prominent theory on the function of alpha oscillations is that they reflect a mechanism by which brain regions that represent task-irrelevant information are suppressed in order to prioritize task-relevant information processing (Jensen \& Mazaheri, 2010; Kelly et al., 2006; Klimesch et al., 2007; also see, Palva \& Palva, 2007 for an alternative account of alpha). Specifically, alpha oscillations have been consistently observed in several sensory modalities during WM maintenance. Despite this consistent involvement, the functional interpretation of alpha is still debated. Some studies argue that delay period alpha represents functional inhibition of taskirrelevant brain areas (Jensen et al., 2002; Jokisch \& Jensen, 2007; Medendorp et al., 2007; Sauseng et al., 2009). According to this hypothesis, our result of increased delay period posterior alpha power for Relation trials may indicate that sensory regions are being inhibited when a spatial relation is maintained compared to a spatial location. This inhibition hypothesis interpretation is in line with our previous work (Ikkai et al., 2014), which showed increased posterior alpha power and decreased alpha lateralization when a spatial relation was maintained compared to an item-specific location. Here we modified the task design used previously by Ikkai et al. (2014) in order to directly examine not only the maintenance of abstract information in WM, but the crucial step of transforming a sensory representation into an abstract representation. In addition, by requiring participants to maintain two sensory stimuli in WM over an initial delay and then cuing them to transform those sensory memoranda into either one spatial relation or one spatial location, we were able to ensure that differences between the two trial types were not due to differential attentional states at the time of stimulus encoding.

Extending previous findings, here we also found increased frontal alpha power and frontal-posterior phase synchrony for Relation trials compared to Location trials. It has been previously suggested that alpha phase synchrony between frontal and sensory regions plays a role in the inhibition of anticipated, task-irrelevant stimuli and that frontal alpha is localized to dorsolateral and anterior PFC regions (Bonnefond \& Jensen, 2012; Sadaghiani et al., 2012). Further, it has been suggested that PFC controls posterior alpha modulation (Sauseng et al., 2005; Zanto et al., 2011). However, it is worth noting that these previous studies and ours here utilize PLV as a measure of phase synchrony, which do not provide directionality; although Zanto et al., (2011) used transcranial magnetic stimulation, which does allow for a causal claim about PFC modulating posterior alpha. Nonetheless, our pattern of results are consistent with the idea that sensory cortex may be suppressed when abstract relations are derived and maintained in WM. However, given that we did not introduce overt distractors into our task, as in previous studies of alpha as an inhibitory mechanism (Bonnefond \& Jensen, 2012; Zanto et al., 2011), we cannot directly provide support for the inhibition hypothesis of alpha based on our results. Instead, we assert that abstract, relational information is derived and maintained in WM via distinct neural oscillatory patterns in the alpha frequency band. In addition to suppressing anticipated distracting stimuli, such a mechanism could serve to suppress 
residual memory representations of the now-irrelevant sample stimulus or prepare to control processing of the upcoming test stimulus according to its relational information rather than a sensory match. Future studies will be needed to directly test these possibilities.

Alternatively, others have linked alpha activity directly to the processes underlying WM maintenance, such as top-down control of WM representations (Herrmann, Senkowski, \& Rottger, 2004; Leiberg, Lutzenberger, \& Kaiser, 2006; Palva, Kulashekhar, Hamalainen, \& Palva, 2011; Sauseng et al., 2005). Particularly relevant to the current study, this account rests on evidence that frontal and posterior sites show increased phase synchrony during WM maintenance suggesting that posterior alpha modulation is controlled by PFC (e.g., Sauseng et al., 2005). This idea is consistent with our results here showing that not only is posterior alpha power increased, but frontal-posterior PLV is also greater during Relation trials compared to Location trials. According to this account of alpha, our results may indicate that maintaining a spatial relation requires greater top-down executive control than maintaining a sensory representation. Previous work has shown that greater relational complexity and abstractness of representations are associated with activity in more anterior PFC regions (for a review see, Badre 2008). This notion of a control hierarchy together with our results may suggest that the difference in alpha activity here may be due to the abstract nature of the representation maintained during Relation trials compared to maintaining a concrete spatial location. This idea is speculative given the current data, but future studies could manipulate the degree of concreteness/abstractness of WM information to examine changes in posterior alpha power as well as frontal-posterior phase synchrony.

While our current results cannot differentiate between these two alternative accounts of alpha, we can conclude that WM for concrete, spatial locations and abstract, spatial relations are supported via distinct neural oscillatory patterns. Here we designed our task to require participants to either derive an abstract relationship or a specific spatial location from sensorybased information and then maintain that information in WM across a delay. While our results cannot differentiate between the derivation and manipulation processes, our results do suggest that alpha is playing a differential role in WM for these two types of information.

One potential caveat is that there was a significant difference in accuracy between the two trial types, with participants being more accurate on Relation than Location trials. This could indicate that the Location trials were more difficult than the Relation trials. However, we do not believe this limits any of our EEG data interpretations for several reasons. Accuracy was very high for both trial types $(>90 \%)$ and the low percentage of incorrect trials were not included in the EEG analysis. Also, a correlation analysis revealed no significant relationship between task accuracy and alpha power, which suggests that task difficulty cannot explain the differences shown here. Most importantly, we designed the current task to equate the two trial types as closely as possible regarding WM demands. The crucial manipulation was that participants had to convert or manipulate the initially stored sensory information into either one spatial relation or one spatial location. Regardless of trial type, only one piece of relevant information was maintained during the Delay 2 period equating memory load, which was the primary focus of our analyses. An important future direction will be to examine how various WM loads of Relation versus Location information influence the resulting oscillatory modulations. For example, if increased posterior alpha power is critical for maintenance of spatial relations, then increasing the amount of to-be-remembered relational information may have a parametric effect on power and phase synchrony. While this question is beyond the scope of the current study, future work should target this approach to further elucidate the role of alpha oscillations in maintaining distinct representations in WM.

As in our previous studies, there is no evidence that the differences in maintaining an abstract, spatial relation versus a concrete, spatial location here arise from a differential reliance on a verbal strategy. We have demonstrated that when introducing a verbal load manipulation, whereby letter stimuli were maintained concurrently with the type of tasks used here, performance is not differentially impacted for Relation and Location trials (Ackerman \& Courtney, 2012; Ikkai et al., 2014). Further, Ackerman and Courtney (2012) demonstrated using fMRI that brain areas preferentially activated by Relation trials were distinct from those activated for verbal WM. Together, those results suggest that while participants may have supplemented Relation and Location WM representations with verbal re-coding, they did not do so more for one task than the other. Further, previous work has shown that ignoring verbal information is associated with a left lateralized increase in alpha power (Dube, Payne, Sekuler, \& Rotello, 2013). This finding was in a group of left fronto-temporal electrodes that are consistent with our left frontal cluster that showed greater alpha power for Relation compared to Location trials. Dube et al. (2013) concluded that this increase in left frontal alpha was indicative of ignoring verbal information, which would be in direct opposition to a verbal strategy account here for our Relation trials. Future work should directly investigate how abstract spatial information, like relationships, are related or not to verbal information processing.

Finally, one often cited concern regarding PLV data is the possibility of volume conduction overinflating the phase synchrony estimates. While volume conduction can elicit artificially high PLVs for short-range synchronies, the PLV results of interest presented here represent between region or longrange synchronization (i.e., posterior to frontal), which cannot be explained by volume conduction (Lachaux et al., 1999). Furthermore, we ran our PLV analysis with an SCD transformation and found the same significantly increased posterior- 
frontal alpha phase synchrony for Relation trials compared to Location trials, which suggests that our results are not due to volume conduction.

One unanticipated result that emerged was a difference in alpha power between the two trial types during the Test period. This result was spatially non-specific, as the resulting cluster included most electrodes across the scalp. In addition, this increased alpha power for the Relation trials followed a large spike in alpha phase synchrony that was equivalent in magnitude for the Relation and Location trials. These findings again suggest a greater role for oscillatory activity beyond just suppressing irrelevant sensory inputs. Dynamic control of the magnitude and synchrony of this activity across brain areas may play a general role in the ability to compare current sensory inputs to maintained WM representations. The need in the Relations trials to first convert the test stimulus into a relational representation in order to then compare the two relations in WM may place greater demands on alpha power across entire brain networks. The lack of specificity of our Test period results prohibits any strong conclusions, but future work may be able to test this notion in a more controlled manner.

In conclusion, the current results support the idea that abstract and sensory information are derived and maintained in WM via distinct neural oscillatory patterns. Maintaining abstract information in WM was associated with increased posterior alpha power, which may suggest that when abstract information is relevant, sensory information may interfere and thus may need to be suppressed or filtered out. Moreover, deriving abstract information from a sensory representation appears to rely on a combination of posterior alpha modulation, frontal alpha power modulation and the synchrony of alpha oscillations between these regions. Dynamic changes in alpha power and alpha phase synchrony between posterior and frontal regions seem to play a crucial part in processing abstract versus sensory information and in their selective online maintenance in WM.

Acknowledgments We wish to thank Antonio Vergara for help with data collection and Ajay Pillai for helpful comments and conversations. This work was supported by NIH grants R01 MH082957 and R01 DA13165 to SMC, a K23 NS073626 to JBE, and a Johns Hopkins University Science of Learning Institute Fellowship to KJB.

\section{References}

Ackerman, C. M., \& Courtney, S. M. (2012). Spatial relations and spatial locations are dissociated within prefrontal and parietal cortex. Journal of Neurophysiology, 108(9), 2419-2429. doi:10.1152/jn. 01024.2011

Adrian, E. D., \& Matthews, B. H. (1934). Berger rhythm: Potential changes from occipital lobes in man. Brain, 57, 355-385.

Badre, D. (2008). Cognitive control, hierarchy, and the rostro-caudal organization of the frontal lobes. Trends in Cognitive Sciences, 12(5), 193-200. doi:10.1016/J.Tics.2008.02.004
Bastiaansen, M., Mazaheri, A., \& Jensen, O. (2012). Beyond ERP's: Oscillatory neuronal dynamics. In S. J. Luck \& E. S. Kappenman (Eds.), The Oxford Handbook of event-related potential components. USA: Oxford University Press.

Bengson, J. J., Mangun, G. R., \& Mazaheri, A. (2012). The neural markers of an imminent failure of response inhibition. NeuroImage, 59(2), 1534-1539. doi:10.1016/j.neuroimage.2011. 08.034

Bonnefond, M., \& Jensen, O. (2012). Alpha oscillations serve to protect working memory maintenance against anticipated distracters. Current Biology, 22(20), 1969-1974. doi:10.1016/j.cub.2012. 08.029

Brainard, D. H. (1997). The psychophysics toolbox. Spatial Vision, 10(4), 433-436

Chafee, M. V., Averbeck, B. B., \& Crowe, D. A. (2007). Representing spatial relationships in posterior parietal cortex: Single neurons code object-referenced position. Cerebral Cortex, 17(12), 2914-2932. doi:10.1093/cercor/bhm017

Cohen, J. R., Sreenivasan, K. K., \& D'Esposito, M. (2014). Correspondence between stimulus encoding- and maintenancerelated neural processes underlies successful working memory. Cerebal Cortex, 24(3), 593-599. doi:10.1093/cercor/bhs339

Courtney, S. M., Ungerleider, B. G., Keil, K., \& Haxby, J. V. (1997). Transient and sustained activity in a distributed neural system for human working memory. Nature, 386(6625), 608-611. doi:10. 1038/386608a0

Curtis, C. E., \& D'Esposito, M. (2003). Persistent activity in the prefrontal cortex during working memory. Trends in Cognitive Science, 7(9), 415-423. doi:10.1016/S1364-6613(03)00197-9

D'Esposito, M. (2007). From cognitive to neural models of working memory. Philosophical Transactions of the Royal Society of London. Series B, Biological Sciences, 362(1481), 761-772. doi: 10.1098/rstb.2007.2086

Dube, C., Payne, L., Sekuler, R., \& Rotello, C. M. (2013). Paying attention to attention in recognition memory: Insights from models and electrophysiology. Psychological Science, 24(12), 2398-2408. doi: 10.1177/0956797613492426

Freedman, D. J., Riesenhuber, M., Poggio, T., \& Miller, E. K. (2001). Categorical representation of visual stimuli in the primate prefrontal cortex. Science, 291(5502), 312-316. doi:10.1126/science.291. 5502.312

Fu, K. M., Foxe, J. J., Murray, M. M., Higgins, B. A., Javitt, D. C., \& Schroeder, C. E. (2001). Attention-dependent suppression of distracter visual input can be cross-modally cued as indexed by anticipatory parieto-occipital alpha-band oscillations. Brain Research. Cognitive Brain Research, 12(1), 145-152.

Funahashi, S., Bruce, C. J., \& Goldman-Rakic, P. S. (1989). Mnemonic coding of visual space in the monkey's dorsolateral prefrontal cortex. Journal of Neurophysiology, 61(2), 331-349.

Fuster, J. M. (2000). Executive frontal functions. Experimental Brain Research, 133(1), 66-70.

Genovesio, A., Brasted, P. J., Mitz, A. R., \& Wise, S. P. (2005). Prefrontal cortex activity related to abstract response strategies. Neuron, 47(2), 307-320. doi:10.1016/j.neuron.2005.06.006

Gnadt, J. W., \& Andersen, R. A. (1988). Memory related motor planning activity in posterior parietal cortex of macaque. Experimental Brain Research, 70(1), 216-220.

Goldman-Rakic, P. S. (1995). Cellular basis of working memory. Neuron, 14, 477-485.

Grossman, A., Kronland-Martinet, R., \& Morlet, J. (1989). Reading and understanding continuous wavelets transforms. In J. M. Combes, A. Grossmann, \& P. Tchamitchian (Eds.), Wavelets, time-frequency methods and phase space (pp. 2-20). Berlin: Springer-Verlag.

Herrmann, C. S., Senkowski, D., \& Rottger, S. (2004). Phase-locking and amplitude modulations of EEG alpha: Two measures reflect 
different cognitive processes in a working memory task. Experimental Psychology, 51(4), 311-318. doi:10.1027/16183169.51.4.311

Hummel, F., \& Gerloff, C. (2005). Larger interregional synchrony is associated with greater behavioral success in a complex sensory integration task in humans. Cerebral Cortex, 15(5), 670-678. doi: 10.1093/cercor/bhh170

Ikkai, A., Blacker, K. J., Lakshmanan, B. M., Ewen, J. B., \& Courtney, S. M. (2014). Maintenance of relational information in working leads to suppression of the sensory cortex. Journal of Neurophysiology, 112(8), 1903-1915. doi:10.1152/jn.00134.2014

Jensen, O., Gelfand, J., Kounios, J., \& Lisman, J. E. (2002). Oscillations in the alpha band $(9-12 \mathrm{~Hz})$ increase with memory load during retention in a short-term memory task. Cerebral Cortex, 12(8), 877-882.

Jensen, O., \& Mazaheri, A. (2010). Shaping functional architecture by oscillatory alpha activity: Gating by inhibition. Frontiers in Human Neuroscience, 4, 186. doi:10.3389/fnhum.2010.00186

Jokisch, D., \& Jensen, O. (2007). Modulation of gamma and alpha activity during a working memory task engaging the dorsal or ventral stream. Journal of Neuroscience, 27(12), 3244-3251. doi:10.1523/ JNEUROSCI.5399-06.2007

Kelly, S. P., Lalor, E. C., Reilly, R. B., \& Foxe, J. J. (2006). Increases in alpha oscillatory power reflect an active retinotopic mechanism for distracter suppression during sustained visuospatial attention. Journal of Neurophysiology, 95(6), 3844-3851. doi:10.1152/jn. 01234.2005

Klimesch, W., Doppelmayr, M., Schwaiger, J., Auinger, P., \& Winkler, T. (1999). 'Paradoxical' alpha synchronization in a memory task. Brain Research. Cognitive Brain Research, 7(4), 493-501.

Klimesch, W., Sauseng, P., \& Hanslmayr, S. (2007). EEG alpha oscillations: The inhibition-timing hypothesis. Brain Research Reviews, 53(1), 63-88. doi:10.1016/j.brainresrev.2006.06.003

Krause, C. M., Lang, A. H., Laine, M., Kuusisto, M., \& Porn, B. (1996). Event-related EEG desynchronization and synchronization during an auditory memory task. Electroencephalography and Clinical Neurophysiology, 98(4), 319-326. doi:10.1016/0013-4694(96) 00283-0

Kuo, B.-C., Stokes, M. G., \& Nobre, A. C. (2012). Attention modulates maintenance of representations in visual short-term memory. Journal of Cognitive Neuroscience, 24(1), 51-60. doi:10.1162/ jocn a 00087

Lachaux, J. P., Rodriguez, E., Martinerie, J., \& Varela, F. J. (1999). Measuring phase synchrony in brain signals. Human Brain Mapping, 8(4), 194-208.

Leiberg, S., Lutzenberger, W., \& Kaiser, J. (2006). Effects of memory load on cortical oscillatory activity during auditory pattern working memory. Brain Research, 1120(1), 131-140. doi:10.1016/j.brainres. 2006.08.066

Levy, R., \& Goldman-Rakic, P. S. (2000). Segregation of working memory functions within the dorsolateral prefrontal cortex. Experimental Brain Research, 133(1), 23-32.

Manza, P., Hau, C. L., \& Leung, H. C. (2014). Alpha power gates relevant information during working memory updating. Journal of Neuroscience, 34(17), 5998-6002. doi:10.1523/JNEUROSCI. 4641-13.2014

Maris, E., \& Oostenveld, R. (2007). Nonparametric statistical testing of EEG- and MEG-data. Journal of Neuroscience Methods, 164(1), 177-190. doi:10.1016/j.jneumeth.2007.03.024

Medendorp, W. P., Kramer, G. F., Jensen, O., Oostenveld, R., Schoffelen, J. M., \& Fries, P. (2007). Oscillatory activity in human parietal and occipital cortex shows hemispheric lateralization and memory effects in a delayed double-step saccade task. Cerebral Cortex, 17(10), 2364-2374. doi:10.1093/cercor/bhl145
Montojo, C. A., \& Courtney, S. M. (2008). Differential neural activation for updating rule versus stimulus information in working memory. Neuron, 59(1), 173-182. doi:10.1016/j.neuron.2008.05.012

Nichols, T. E., \& Holmes, A. P. (2002). Nonparametric permutation tests for functional neuroimaging: A primer with examples. Human Brain Mapping, 15(1), 1-25.

Oostenveld, R., Fries, P., Maris, E., \& Schoffelen, J. M. (2011). FieldTrip: Open source software for advanced analysis of MEG, EEG, and invasive electrophysiological data. Computational Intelligence and Neuroscience, 2011, 156869. doi:10.1155/2011/156869

Palva, S., Kulashekhar, S., Hamalainen, M., \& Palva, J. M. (2011). Localization of cortical phase and amplitude dynamics during visual working memory encoding and retention. Journal of Neuroscience, 31(13), 5013-5025. doi:10.1523/JNEUROSCI.5592-10.2011

Palva, S., \& Palva, J. M. (2007). New vistas for alpha-frequency band oscillations. Trends in Neuroscience, 30(4), 150-158. doi:10.1016/j. tins.2007.02.001

Pelli, D. G. (1997). The VideoToolbox software for visual psychophysics: Transforming numbers into movies. Spatial Vision, 10(4), 437-442.

Percival, D., \& Walden, A. (1993). Spectral analysis for physical applications. Cambridge: Cambridge University Press.

Perrin, F., Pernier, J., Bertrand, O., \& Echallier, J. F. (1989). Spherical splines for scalp potential and current density mapping. Electroencephalography and Clinical Neurophysiology, 72(2), 184-187.

Pfurtscheller, G., Stancak, A., Jr., \& Neuper, C. (1996). Event-related synchronization (ERS) in the alpha band-an electrophysiological correlate of cortical idling: A review. International Journal of Psychophysiology, 24(1-2), 39-46.

Rihs, T. A., Michel, C. M., \& Thut, G. (2007). Mechanisms of selective inhibition in visual spatial attention are indexed by alpha-band EEG synchronization. European Journal of Neuroscience, 25(2), 603 610. doi:10.1111/j.1460-9568.2007.05278.x

Roux, F., \& Uhlhaas, P. J. (2013). Working memory and neural oscillations: Alpha-gamma versus theta-gamma codes for distinct WM information? Trends in Cognitive Sciences. doi:10.1016/j.tics. 2013.10.010

Rutman, A., Clapp, W. C., Chadick, J. Z., \& Gazzaley, A. (2010). Early top-down control of visual processing predicts working memory performance. Journal of Cognitive Neuroscience, 22(6), 12241234. doi:10.1162/jocn.2009.21257

Sadaghiani, S., Scheeringa, R., Lehongre, K., Morillon, B., Giraud, A. L., D'Esposito, M., \& Kleinschmidt, A. (2012). alpha-band phase synchrony is related to activity in the fronto-parietal adaptive control network. Journal of Neuroscience, 32(41), 14305-14310. doi:10. 1523/JNEUROSCI.1358-12.2012

Sauseng, P., Klimesch, W., Heise, K. F., Gruber, W. R., Holz, E., Karim, A. A.,...Hummel, F. C. (2009). Brain oscillatory substrates of visual short-term memory capacity. Current Biology, 19(21), 1846-1852. doi: 10.1016/j.cub.2009.08.062

Sauseng, P., Klimesch, W., Stadler, W., Schabus, M., Doppelmayr, M., Hanslmayr, S.,...Birbaumer, N. (2005). A shift of visual spatial attention is selectively associated with human EEG alpha activity. The European Journal of Neuroscience, 22(11), 2917-2926. doi: 10. $1111 / \mathrm{j} .1460-9568.2005 .04482 . x$

Serences, J. T., Ester, E. F., Vogel, E. K., \& Awh, E. (2009). Stimulusspecific delay activity in human primary visual cortex. Psychological Science, 20(2), 207-214. doi:10.1111/j.1467-9280. 2009.02276.x

Smith, E. E., \& Jonides, J. (1999). Storage and executive processes in the frontal lobes. Science, 283(5408), 1657-1661. doi:10.1126/Science. 283.5408.1657

Swaminathan, S. K., \& Freedman, D. J. (2012). Preferential encoding of visual categories in parietal cortex compared with prefrontal cortex. Nature Neuroscience, 15(2), 315-320. doi:10.1038/nn.3016 
Todd, J. J., \& Marois, R. (2004). Capacity limit of visual short-term memory in human posterior parietal cortex. Nature, 428(6984), 751-754. doi:10.1038/nature02466

Tsujimoto, S., Genovesio, A., \& Wise, S. P. (2012). Neuronal activity during a cued strategy task: Comparison of dorsolateral, orbital, and polar prefrontal cortex. Journal of Neuroscience, 32(32), 11017-11031. doi:10.1523/JNEUROSCI.1230-12.2012

Ungerleider, L. G., Courtney, S. M., \& Haxby, J. V. (1998). A neural system for human visual working memory. Proceedings of the National Academy of Sciences of the United States of America, 95(3), 883-890.

van Dijk, H., van der Werf, J., Mazaheri, A., Medendorp, W. P., \& Jensen, O. (2010). Modulations in oscillatory activity with amplitude asymmetry can produce cognitively relevant event-related responses. Proceedings of the National Academy of Sciences of the United States of America, 107(2), 900-905. doi:10.1073/pnas.0908821107

van Gerven, M., Bahramisharif, A., Heskes, T., \& Jensen, O. (2009). Selecting features for BCI control based on a covert spatial attention paradigm. Neural Networks, 22(9), 1271-1277. doi:10.1016/j. neunet.2009.06.004

Vogel, E. K., McCullough, A. W., \& Machizawa, M. G. (2005). Neural measures reveal individual differences in controlling access to working memory. Nature, 438(24), 500-503. doi:10.1038/nature04171

Wallis, J. D., Anderson, K. C., \& Miller, E. K. (2001). Single neurons in prefrontal cortex encode abstract rules. Nature, 411(6840), 953-956. doi: $10.1038 / 35082081$

Worden, M. S., Foxe, J. J., Wang, N., \& Simpson, G. V. (2000). Anticipatory biasing of visuospatial attention indexed by retinotopically specific alpha-band electroencephalography increases over occipital cortex. Journal of Neuroscience, 20(6), RC63.

Zanto, T. P., Rubens, M. T., Thangavel, A., \& Gazzaley, A. (2011). Causal role of the prefrontal cortex in top-down modulation of visual processing and working memory. Nature Neuroscience, 14(5), 656661. doi:10.1038/nn.2773 